\title{
EDUCAÇÃO PERMANENTE EM SAÚDE COMO PRÁTICA AVALIATIVA AMISTOSA À INTEGRALIDADE EM TERESÓPOLIS, RIO DE JANEIRO
}

\author{
PERMANENT EDUCATION IN HEALTH AS AN EVALUATIVE PRACTICE FRIENDLY TO \\ COMPLETENESS IN TERESÓPOLIS, RIO DE JANEIRO, BRAZIL
}

\author{
EDUCACIÓN PERMANENTE EN SALUD COMO PRÁCTICA EVALUADORA AMIGABLE A \\ LA INTEGRALIDAD EN TERESÓPOLIS, RÍO DE JANEIRO, BRASIL
}

Thaís Sayuri Yamamoto
Mônica Tereza Christa Machado ${ }^{2}$
Aluísio Gomes da Silva Junior

Resumo O objetivo deste artigo é discutir o papel da educação permanente em saúde como prática avaliativa amistosa à integralidade no cotidiano dos serviços de saúde, além de sua influência na mudança do processo de trabalho das equipes de saúde da família, tomando como exemplo a experiência do município de Teresópolis, no Rio de Janeiro, em 2007 e 2008. Realizou-se uma pesquisa qualitativa de abordagem descritiva. A base teórica compôs-se de revisão bibliográfica, análise de documentos oficiais, atas das reuniões dos facilitadores com a coordenadora do grupo e atas da Comissão Intergestores Bipartite. O campo de observação consistiu de entrevista semiestruturada com o grupo de facilitadores. Para o estudo das entrevistas e atas, optou-se pela análise de conteúdo e efetuou-se o cotejamento das fontes como prova eficiente de validação. Percebeu-se que os profissionais ainda têm dificuldade em reconhecer como ferramentas de avaliação instrumentos que não os oficiais. No entanto, em Teresópolis, a prática de educação permanente foi capaz de promover mudanças no processo de trabalho, viabilizar formação crítica e reflexiva dos atuais e futuros profissionais de saúde, fortalecer a participação social e aproximar a gestão das questões locais de saúde, comprovando que pode ser considerada uma prática avaliativa amistosa à integralidade.

Palavras-chave processo de trabalho; educação permanente em saúde; prática avaliativa amistosa à integralidade.
Abstract The purpose of this article is to discuss the role continuing education in health plays as a friendly evaluative practice for completeness in the daily life of health services, as well as its influence in changing the family health teams' working process. It is based on the experience of the municipality of Teresópolis, state of Rio de Janeiro, Brazil, in 2007 and 2008. A qualitative survey with a descriptive approach was conducted. The theoretical basis consisted of a review of the literature, of the analysis of official documents, minutes of the meetings of the facilitators with the coordinator of the group, and the minutes of the Bipartite Inter-Management Commission. The field of observation consisted of a semi-structured interview with the group of facilitators. To analyze the interviews and minutes, we opted for content analysis and compared the sources as an efficient proof of validation. It was noticed that professionals still find it hard to recognize instruments other than the official ones as assessment tools. However, in Teresópolis, the practice of continuing education was able to promote changes in the labor process, enabling critical and reflective training among current and future health professionals, strengthening social participation and bring management closer to the local health issues, thus proving that a friendly evaluative practice can be considered for completeness. Keywords work process; continuing education in health; evaluative practice friendly to completeness. 


\section{Introdução}

Na atenção básica, mas principalmente nas unidades vinculadas à Estratégia Saúde da Família (ESF), o trabalho em saúde deve se configurar como um trabalho de grupo. Porém, algumas vezes, os profissionais realizam tarefas justapostas, formando o que Peduzzi (2006) denomina de 'equipe agrupamento', contrapondo-se à noção de 'equipe integração', definida como a equipe em que ocorre a articulação das ações e a interação dos agentes.

Aliada à fragmentação do processo de trabalho, verifica-se uma formação para a saúde também de modo fragmentado, gerando especialistas cujo conhecimento técnico específico se sobrepõe ao conhecimento integral das realidades locais e ao conhecimento de outros saberes (Ceccim, 2005a).

Mascarenhas (2003) afirma a importância e a necessidade da atuação de toda a equipe na resposta assistencial, valorizando-se o conjunto da equipe no fazer saúde. Todavia, pontua que, para tal, é preciso estruturar a capacitação técnica e reciclar os profissionais envolvidos. Esta ideia é reforçada por L'Abbate (2004), para quem os processos de capacitação devem ser construídos de modo a criar condições, as mais favoráveis possíveis, para permitir a construção de sujeitos autônomos e críticos. Por sua vez, Faria e colaboradores (2009) afirmam que a avaliação crítica de todo o processo de trabalho e sua execução levam à construção do conhecimento e de novas capacidades que culminam no desenvolvimento do próprio processo de trabalho.

É nesse contexto, portanto, de formação/avaliação do processo de trabalho, que veio se inserir a Política Nacional de Educação Permanente em Saúde (PNEPS), instituída pela portaria n. 198/GM/MS, em 13 de fevereiro de 2004, proposta de prática pedagógica que coloca o cotidiano do trabalho em saúde como central aos processos educativos, ao mesmo tempo que o coloca sob problematização, isto é, em autoanálise e autogestão (Brasil, 2004b; Ceccim, 2005b).

A educação permanente é a realização do encontro entre o mundo de formação e o mundo do trabalho, onde o aprender e o ensinar se incorporam ao cotidiano das organizações e ao trabalho (Brasil, 2004a, p. 10).

O texto da Politica nacional para formação e desenvolvimento para o SUS: caminhos para a educação permanente propõe que os processos de qualificação dos trabalhadores da saúde tomem como referência as necessidades de saúde das pessoas e das populações, da gestão setorial e do controle social em saúde. Que tenham como objetivos a transformação das práticas profissionais e da própria organização do trabalho e sejam estruturados a partir da problematização da atuação e da gestão setorial em saúde (Brasil, 2004a). 
Para Ceccim e Ferla (2008), a educação permanente em saúde (EPS) não é só um processo didático-pedagógico; é um processo político-pedagógico que trata de mudar o cotidiano do trabalho na saúde e de colocar o cotidiano profissional em invenção viva, em equipe e com os usuários. Contudo, para avaliar o processo de trabalho de uma equipe de saúde da família, Silva Junior e Mascarenhas (2004) verificaram que a avaliação normativa, a qual prioriza aspectos como produção de serviços e indicadores de impacto epidemiológico, não alcança a captação de aspectos subjetivos inerentes à avaliação, como as relações com os usuários e a integralidade em saúde.

Felisberto $(2004,2006)$ destaca que o fomento de uma cultura avaliativa na atenção primária pode ampliar as possibilidades de os usuários obterem o cuidado que desejam ou esperam, auxiliar os gestores na formulação de políticas e na tomada de decisão - e também os trabalhadores quanto à reflexão sobre suas práticas de saúde.

Partindo desses referenciais, Pinheiro e Silva Junior (2008), apoiados em Ayres (2004), propõem uma noção de práticas como ações exercidas cotidianamente nos serviços, como referenciais ricos de análise e intervenção que não se esgotam nas ferramentas tecnológicas já existentes, nem se pretendem tornar técnicas a serem compartilhadas. Ao considerarem a integralidade da atenção à saúde, esclarecem que

as práticas avaliativas na atenção básica passam a abrigar as ações efetivas dos atores nas situações de encontro com a alteridade, ou seja, com a diversidade e a pluralidade humana. Significa ir além da execução de técnicas, convivendo e, por isso, sendo definidas por experiências humanas e singulares (Pinheiro e Silva Junior, 2008, p. 28).

Assim, o objetivo deste artigo foi refletir sobre o papel da EPS como prática avaliativa, enfocando sua porosidade com a discussão da integralidade como eixo estruturador de ações e serviços de saúde, além de sua influência na mudança do processo de trabalho das equipes. Para tanto, utilizaremos o exemplo da institucionalização das ações do grupo de EPS nas unidades inseridas na ESF de Teresópolis para ilustrar e demonstrar de forma empírica essa prática.

Embora a institucionalização da PNEPS tenha acontecido em 2004, com a portaria n. 198/GM/MS (Brasil, 2004b), e suas diretrizes estabelecidas em 2007, com a portaria n. 1.996/GM/MS (Brasil, 2007), poucos municípios aderiram e conceberam experiências de educação permanente em seus serviços de saúde. Somente em setembro de 2011 foram definidos os recursos financeiros do Ministério da Saúde (MS) para essa política - portaria n. 2.200/GM/MS (Brasil, 2011) -, o que reavivou as discussões sobre a EPS e deu novo impulso à adesão de outros municípios. 
Logo, analisar a implantação das práticas de EPS em Teresópolis, nos anos de 2007 e 2008, sobretudo no que concerne às mudanças provocadas no processo de trabalho, pode contribuir não só para subsidiar outros municípios no processo de implementação da PNEPS como também para levantar críticas que levem a mudanças na própria política.

Antes disso, porém, é necessário contextualizar e integrar os conceitos explorados nesta pesquisa.

\section{O processo de trabalho e a EPS}

O conceito 'processo de trabalho em saúde' diz respeito à dimensão microscópica do cotidiano do trabalho em saúde, à prática dos trabalhadores/profissionais de saúde inseridos no dia a dia da produção e consumo de serviços de saúde (Peduzzi e Schraiber, 2008).

Para Silva e Peduzzi (2011), o trabalho em saúde é caracterizado pela intersubjetividade nas relações entre trabalhadores-usuários e entre os trabalhadores - e também pela imprevisibilidade e incerteza, relacionadas à variedade de eventos e complexidades presentes no processo saúde-doença.

Faria e colaboradores (2009) propõem que, de maneira geral, os componentes dos processos de trabalho são, eles próprios, produtos de trabalho. A sua produção é uma criação humana consciente, uma adequação concreta de recursos existentes aos fins postos. Por conseguinte, o funcionamento reiterativo dos processos de trabalho implica algum tipo de avaliação e crítica que incide sobre os procedimentos e conhecimentos de cada processo de trabalho, possibilitando a sua transformação consciente.

Os componentes do processo de trabalho, incluindo seus fins, sua execução e sua própria avaliação, sofrem modificações a partir desse processo crítico de avaliação, ou seja, os processos de trabalho são sempre avaliados em função dos seus resultados, mais ou menos intensos, consciente e sistematicamente, conforme as condições sociais e institucionais em que eles se exercem (Faria et al., 2009, p. 27).

Assim, conclui-se que a avaliação deve ser parte do próprio processo de trabalho em saúde, gerando mudanças em suas dimensões de produtividade, satisfação e realização pessoal dos profissionais e dos usuários, economia de meios e otimização de recursos, de modo a adequá-lo à realidade na qual está inserido.

Partindo-se do princípio de que o trabalho em saúde é realizado por 'homens' e, segundo Paulo Freire (2007, p. 31), “o homem se identifica com sua própria ação: objetiva o tempo, temporaliza-se, faz-se homem-história", nessa relação entre 'homem' (trabalhador da saúde) e campo da ação (setor saúde) cabe a compreensão de que 
o trabalhador social que atua numa realidade, a qual, mudando, permanece para mudar novamente, precisa saber que, como homem, somente pode entender ou explicar a si mesmo como um ser em relação com esta realidade; que seu 'quefazer' nesta realidade se dá com outros homens, tão condicionados como ele pela realidade dialeticamente permanente e mutável e que, finalmente, precisa conhecer a realidade na qual atua com outros homens (Freire, 2007, p. 48).

Essa visão permite entender como a EPS se articula com o processo de trabalho em saúde, uma vez que se apoia no conceito de ensino problematizador e de aprendizagem significativa - isto é, ensino-aprendizagem embasado na produção de conhecimentos que respondam a perguntas que pertencem ao universo de experiências e vivências de quem aprende e que gerem novas perguntas sobre o ser e o atuar no mundo (Ceccim e Ferla, 2008).

A EPS reconhece o caráter educativo do próprio trabalho, que passa a ser compreendido não apenas em seu sentido instrumental da produção de resultados, da ação dirigida a um dado fim já definido a priori, mas também como espaço de problematização, reflexão, diálogo e construção de consensos por meio dos quais se torna possível promover mudanças e transformações na perspectiva da integralidade da saúde (Ceccim, 2005a, 2005b; Ceccim e Feuerwerker, 2004; Peduzzi et al., 2009).

Todavia, para se compreender a EPS, é necessário pensar além do conceito de educação e de EPS propriamente dito, enveredando-se pelo campo da saúde, com suas particularidades, complexidades e relações. Espera-se que sua prática no cotidiano dos serviços viabilize a avaliação crítica de um processo de trabalho que é, portanto, intersubjetivo, imprevisível e em constante reconstrução, possibilitando a melhora da assistência em saúde para todos os atores envolvidos: profissionais, usuários e gestores.

Ademais, Ceccim (2005b) assinala que o conceito de EPS foi adotado pelo MS com o objetivo de tornar a rede pública de saúde uma rede de ensino-aprendizagem no exercício do trabalho - não no prolongamento do tempo ou carreira dos trabalhadores, mas na ampla intimidade entre formação, gestão, atenção e participação nessa área específica de saberes e de práticas, mediante as intercessões promovidas pela educação na saúde.

Entretanto, para constituir o Sistema Único de Saúde (SUS) verdadeiramente como uma rede-escola, há necessidade de descentralizar e disseminar capacidade pedagógica por dentro do setor, isto é, entre seus trabalhadores; entre os gestores de ações, serviços e sistemas de saúde; entre trabalhadores e gestores com os formadores e entre trabalhadores, gestores e formadores com o controle social em saúde (Ceccim, 2005b).

A EPS dos profissionais deve constituir parte do pensar e do fazer dos trabalhadores, com a finalidade de propiciar o crescimento pessoal e profissional destes, bem como contribuir para a organização do processo de trabalho, 
por meio de etapas que venham a problematizar a realidade e produzir mudanças que possam fomentar o alcance ou a aproximação dos objetivos de universalização, integralidade e equidade, ao mesmo tempo qualificando a atenção à saúde (Lino et al., 2009).

Como política nacional, a EPS visaria, então, à melhora do profissional de saúde por meio do incremento de suas habilidades técnicas e relacionais e da otimização de seu processo de trabalho. O desenvolvimento da qualidade do atendimento prestado aos usuários seria consequência da priorização da educação dos profissionais, e não o objetivo final da política.

A EPS, incorporada ao cotidiano da gestão setorial e da condução gerencial dos serviços de saúde, colocaria o SUS como um interlocutor nato das instituições formadoras, na formulação e implementação dos projetos político-pedagógicos de formação profissional, e não mero campo de estágio ou aprendizagem prática (Ceccim e Feuerwerker, 2004).

\section{Práticas avaliativas amistosas à integralidade}

Para Silva (2010), a apreensão de questões relativas ao SUS - em seus princípios de universalidade de acesso, integralidade na atenção à saúde, participação social, preservação da autonomia das pessoas na defesa de sua integridade física e moral, entre outros -, assim como de suas políticas e programas, requer abordagens avaliativas com diferentes matizes. Tais abordagens devem extrapolar a hegemonia positivista, propondo outros olhares, outros referenciais de análise, outros arranjos metodológicos, com a reflexão de noções de participação, emancipação e democracia.

Segundo Pinheiro e Silva Junior (2008), a avaliação pode ser uma ferramenta potente para lograr a participação social na efetivação do direito à saúde como um direito humano, uma vez que, pautada pelo princípio da integralidade, a rede de conhecimentos produzida pelos processos avaliativos pode se voltar para a compreensão das necessidades da população expressas em demandas sociais, levando, consequentemente, à adequação da oferta de serviços. Desse modo, ao acrescentar a centralidade do usuário nos desenhos avaliativos, os autores definem como práticas avaliativas amistosas à integralidade

os diferentes modos de avaliar que tomam como base as ações exercidas cotidianamente nos serviços, na medida em que forjam acoplamentos de saberes e práticas dos sujeitos implicados (gestor, trabalhador e usuário) na produção do cuidado em saúde, subsidiando sua avaliação como intervenção; não se esgotando nas ferramentas avaliativas já existentes e podendo se tornar técnicas não-compartilháveis (Pinheiro e Silva Junior, 2008, p. 28). 
Tais práticas são portadoras de alguns atributos que as tornam mais permeáveis às práticas de integralidade: resultam de interações democráticas entre atores em suas práticas no cotidiano, na relação entre demanda e oferta de produção de cuidado na saúde; garantem a inclusão dos usuários na definição de suas necessidades e na tomada de decisão sobre a oferta de alternativas de cuidado; e possuem elevada potência formativa, capaz de produzir conhecimentos que geram novos valores para juízos, que elaboram respostas qualificadas às necessidades e o modo mais adequado de provê-las.

Os autores citados delimitam, ainda, três dimensões que permitem reconhecer os atributos habilitadores de uma prática avaliativa amistosa à integralidade: a da gestão e organização dos serviços; a das práticas dos trabalhadores; e a do direito à saúde.

Na dimensão da gestão e reorganização dos serviços de saúde, demanda, necessidade e oferta assumem papel fundamental como categorias temáticas transversais - não mais sob um prisma dicotômico, que tende a relacionar a demanda apenas aos usuários ou consumidores, e a oferta somente aos serviços propriamente ditos, mas como conceitos estruturantes, interativos e relacionais, quando vistos pela ótica do cotidiano dos serviços (Pinheiro, 2001).

Nesse sentido, Pinheiro e Silva Junior (2008) propõem que o modo concreto de articular demanda, necessidade e oferta, dizendo-as integrais na atenção e no cuidado, requer a construção coletiva de ferramentas e tecnologias inovadoras no cotidiano das práticas de gestão em saúde, voltadas para a negociação de diferentes pactos e acordos entre instâncias da política setorial e a sociedade civil. Segundo os autores, a criação dessas chamadas 'tecnologias participativas de gestão' configura desenhos organizadores da integralidade em saúde que são constitutivos e constituintes de novas práticas de organização dos serviços de saúde, em que devem ser consideradas

as estratégias para a regulação do acesso às ações e serviços de saúde; a utilização da educação permanente como dispositivo para fortalecer a gestão e a organização da atenção; a utilização de tecnologias participativas no desenho dos processos de trabalho; a permeabilidade ao controle pela sociedade na definição de prioridades etc.; a utilização integrada de sistemas de informação e o reconhecimento das características do território de referência (Pinheiro e Silva Junior, 2008, p. 30-31).

Na segunda dimensão, denominada de conhecimentos e práticas dos trabalhadores, os autores criticam a matriz hegemônica de organização das práticas em saúde - que provoca, no processo de trabalho, uma forma de anomia cultural dos trabalhadores implicados na produção do cuidado - e identificam a capacidade dos trabalhadores de produzir práticas cuidadoras com acolhimento e desfragmentação do atendimento prestado. 
Constatamos, em nossos estudos, que desta forma é possível conferir maior horizontalidade às relações entre seus participantes - gestores, profissionais e usuários do sistema de saúde, no que concerne à autoria de novos conhecimentos a partir das práticas dos sujeitos implicados no cuidado em saúde (Pinheiro e Silva Junior, 2008, p. 31).

O olhar passa a se centrar sobre as concepções e práticas dos atores (trabalhadores, gestores e usuários) no interior dos sistemas de saúde e seus efeitos na atenção básica, recomendando a sua qualificação e EPS.

A terceira dimensão das práticas avaliativas amistosas à integralidade, denominada de direito à saúde, assume caráter fundamental como estratégia para aumentar o grau de sua aceitação e, mais que isso, de sua utilidade, ao enfatizar a centralidade do usuário no processo avaliativo como portador de experiências com alta potência formativa, qualificando-o como avaliador.

Pinheiro e Silva Junior (2008) assumem que é necessário avaliar para afirmar o direito à saúde naquilo que ele tem de mais legítimo: a democratização das informações, com a explicitação dos julgamentos e tomadas de decisões sobre os cuidados oferecidos.

Nesse contexto, o caráter formativo da avaliação ganha expressão e riqueza, na medida em que a incorporação dos conhecimentos acerca das experiências dos usuários pode potencializar a capacidade avaliativa no processo de institucionalização, auxiliando nas transformações das práticas dos gestores, trabalhadores e avaliadores.

\section{A EPS como prática avaliativa amistosa à integralidade}

Para Ceccim (2005b), a percepção, a vivência e a admissão, pelos sujeitos, dos desconfortos existentes relacionados às suas práticas de saúde é que podem, efetivamente, promover mudanças no processo de trabalho. Nesse contexto, a EPS se apresenta como objeto de transformação do processo de trabalho, referida como educação no trabalho, pelo trabalho e para o trabalho nos diversos serviços, cuja finalidade é melhorar a saúde da população (Pinto et al., 2008).

Assim, na dimensão da gestão e reorganização dos serviços de saúde, ao afirmarem que os conhecimentos e práticas dos trabalhadores constituem uma fonte viva de conhecimento para o desenvolvimento de tecnologias de avaliação da atenção básica a serem produzidas nos municípios e para eles de modo que sejam capazes de fortalecer as ações integrais e as práticas avaliativas -, Pinheiro e Silva Junior (2008) legitimam esse aspecto da EPS como atributo de uma prática avaliativa inovadora e amistosa à integralidade.

Na segunda dimensão, de conhecimentos e práticas dos trabalhadores, a EPS é apresentada peremptoriamente como meio de (res)significar os perfis 
de atuação dos profissionais de saúde, assim como dos gestores e usuários, rumo ao fortalecimento da atenção à saúde no SUS.

A dimensão do direito à saúde, por sua vez, reitera um aspecto de relevância na PNEPS: a responsabilidade no processo de produção do cuidado nas respostas às demandas e necessidades geradas pela busca de cuidado pelo usuário, como busca de direitos.

Em suma, a PNEPS ressalta a importância dos seguintes aspectos: articulação entre ensino, trabalho e cidadania; vinculação entre formação, gestão setorial, atenção à saúde e participação social; construção da rede do SUS como espaço de educação profissional; reconhecimento de bases locorregionais como unidades político-territoriais, onde estruturas de ensino e de serviços devem se encontrar em cooperação para a formulação de estratégias para o ensino, assim como para o crescimento da gestão setorial, a qualificação da organização da atenção em linhas de cuidado, o fortalecimento do controle social e o investimento na intersetorialidade (Ceccim e Ferla, 2008).

Conclui-se então que sua inserção nas três dimensões permite dizer que, em teoria, a EPS reúne os atributos necessários para ser considerada uma prática avaliativa amistosa à integralidade, capaz de congregar gestão, formação, práticas de saúde e controle social na adequação da oferta de serviços e consequente efetivação dos princípios do SUS.

Contudo, é preciso determinar se, no cotidiano dos serviços de saúde, a prática da EPS concretiza esses atributos, questão que esta pesquisa pretendeu responder para a realidade do município de Teresópolis.

\section{Notas metodológicas}

Este artigo é um recorte de dissertação de mestrado 4 que teve como objetivo estudar a experiência das práticas de EPS no município de Teresópolis, no estado do Rio de Janeiro, na perspectiva dos facilitadores de educação permanente (Yamamoto, 2011).

A metodologia escolhida foi a de uma pesquisa exploratória-descritiva com base documental e abordagem qualitativa, que utilizou como estratégia o estudo de caso (Yin, 2005; Minayo, 2008) e como modalidade de tratamento analítico a análise de conteúdo (Bardin, 2010).

A base teórica se compôs de revisão bibliográfica, análise de documentos oficiais do município de Teresópolis, das atas das reuniões dos facilitadores com a coordenadora do grupo de facilitadores e das atas da Comissão Intergestora Bipartite do Rio de Janeiro (CIB/RJ). O campo de observação foi formado por entrevista semiestruturada com os facilitadores de EPS.

O questionário de entrevista com os facilitadores de EPS foi elaborado com perguntas semiabertas, desenvolvido com base nas categorias de análise 
previamente definidas: concepções dos facilitadores sobre a integralidade; EPS como dispositivo de mudança do processo de trabalho; e EPS como prática avaliativa amistosa à integralidade no cotidiano dos serviços de saúde.

Na pré-análise do material, fez-se a leitura flutuante das falas dos entrevistados e das atas com o objetivo de identificar as ideias correspondentes às categorias de análise. A exploração do material consistiu em fazer recortes de trechos e frases que representassem unidades de registro, posteriormente agrupadas nas categorias escolhidas. Para o tratamento dos resultados obtidos e a interpretação, foram trabalhados os significados em lugar das inferências estatísticas.

Por fim, efetuou-se o cotejamento das fontes (entrevistas, atas, documentos do município, revisão bibliográfica) como prova eficiente de validação, de modo a valorizar tanto a crítica intersubjetiva como a comparação.

Como participaram desse grupo apenas mulheres (coordenadora e facilitadoras), a partir de agora utilizaremos o termo facilitadoras, no feminino, para designar essas profissionais.

Esta pesquisa foi orientada pelos preceitos da resolução n. 196/96 do Conselho Nacional de Saúde, tendo sido aprovada pelo Comitê de Ética em Pesquisa do Hospital Universitário Antônio Pedro, da Universidade Federal Fluminense, em outubro de 2010 (CEP CMM/Huap n. 250/210, CAAE n. 4797.0.000.258-10). Não contou com financiamento de instituição pública ou privada, e não existiram conflitos de interesse.

\section{Prática da EPS no município}

O município de Teresópolis se localiza na região serrana do estado do Rio de Janeiro, a 87 quilômetros da capital. A projeção demográfica do Instituto Brasileiro de Geografia e Estatística (IBGE) para 2007 foi de uma população estimada em 152.860 habitantes, distribuída prioritariamente em área urbana (84\%), segundo dados coletados no Caderno de Informações em Saúde, do Datasus (2009).

Uma das particularidades desse município é ter um histórico de longa data de parceria entre sua Secretaria Municipal de Saúde (SMS) e o Centro Universitário Serra dos Órgãos (Unifeso), principalmente a partir de agosto de 2005, quando esse convênio foi ampliado, fazendo surgir a Interação Ensino-Trabalho-Comunidade (IETC) - estrutura responsável pela inserção dos estudantes de medicina, enfermagem, odontologia, fisioterapia e psicologia como estagiários nas unidades locais de saúde.

A proposta de integrar ensino, pesquisa e serviços de saúde vem se consolidando no Brasil, nos últimos trinta anos, como fruto do movimento de redemocratização do país e do movimento de reforma sanitária brasileira. 
Pode ser considerada estratégica para aperfeiçoar os modelos de formação, de educação permanente e de gestão do conhecimento na saúde, na construção de sistemas de saúde-escola (Ellery, Bosi e Loiola, 2013).

A convivência dos alunos no cotidiano das unidades de saúde da família (USFs) pode ter contribuído para a decisão da Unifeso, em parceria com a SMS de Teresópolis, de implantar o projeto de EPS no âmbito da IETC, na medida em que esses alunos passaram a encaminhar questões críticas quanto às dificuldades enfrentadas pelas equipes.

Concomitantemente, já havia ações de EPS informais no município por meio da supervisão técnica para especialidades de pediatria, ginecologia e obstetrícia e de clínica geral, que procurava trabalhar nessa lógica com os profissionais tanto das unidades básicas tradicionais quanto das USFs (Pinheiro e Silva Junior, 2009). Todavia, o conteúdo normatizador da PNEPS só foi oficialmente trabalhado no município após a implantação do Polo de Educação Permanente em Saúde na Região Serrana (PEPS-RS), em 2004, em decorrência da portaria GM/MS n. 198 (Alves, 2007).

O início das atividades do grupo de EPS em Teresópolis se deu em fevereiro de 2007, quando profissionais contratadas pela Unifeso e atuantes na rede de saúde municipal foram inseridas nas USFs, caracterizando o que se considerou nesta pesquisa como atividade formal de EPS no município.

Entretanto, por meio da análise das atas da CIB/RJ e do Relatório de Gestão do Município de Teresópolis de 2008, concluiu-se que a instituição da prática da EPS em Teresópolis foi parte de uma iniciativa da IETC, ou seja, da parceria entre a SMS e a Unifeso, inspirada na PNEPS, mas sem o financiamento do MS para tanto. Presume-se que esse fato, aliado à troca dos gestores de saúde com as eleições de 2007, tenha levado ao encerramento do grupo no final de 2008.

\section{Caracterização e funcionamento do grupo de facilitadoras}

O grupo de EPS foi formado por uma coordenadora e quatro facilitadoras, cada uma responsável por três USFs, cobrindo o total das 12 unidades que funcionavam de acordo com a ESF, em Teresópolis, nos anos de 2007 e 2008.

A carga horária acordada com as facilitadoras era de 12 horas semanais, que deveriam ser distribuídas entre visitas às três unidades em que cada uma era responsável e uma reunião semanal com a coordenação.

Nessas reuniões, além das discussões pertinentes ao cotidiano de trabalho nas USFs, a coordenadora propunha alguns textos para serem lidos e discutidos com as facilitadoras, com o propósito de refletir sobre o seu papel e os fundamentos da EPS, além de melhorar o impacto de sua atuação juntamente com as equipes. Embora fossem sempre convidados a participar das 
reuniões, apenas algumas contaram com a participação de gestores do município, do coordenador do internato e dos coordenadores dos cursos de medicina e de enfermagem da Unifeso.

As facilitadoras tinham liberdade para definir se o seu tempo nas unidades seria gasto em reuniões com as equipes; reuniões com as equipes, os alunos inseridos nas unidades e os representantes dos usuários; ou observação participante do processo de trabalho nas USFs para identificação de entraves e posterior discussão. Nas unidades em que havia um conselho gestor, este era convidado a participar das reuniões com as facilitadoras, as equipes e os alunos de graduação da Unifeso, o que demonstra preocupação com a inserção dos usuários na discussão do processo de trabalho das USFs, aspecto fundamental para a integralidade de uma ação.

É preciso ressaltar que, embora o conselho gestor seja uma das formas institucionalizadas de participação social no nível local, trata-se de um espaço de representação dos usuários e não de uma arena de exercício da democracia direta (Vianna, Cavalcanti e Pina Cabral, 2009). Seus membros, mesmo podendo ser considerados como usuários privilegiados, não contam com o que Coelho (2012) elencou como alguns dos dilemas e desafios para a efetiva participação social no SUS: disponibilidade de tempo, transporte, assessoria institucional - enfim, toda uma infraestrutura que geralmente está presente para a participação dos demais segmentos.

Como a proposta das facilitadoras era discutir o processo de trabalho nas equipes e buscar meios de oferecer melhor qualidade de saúde à população, é natural que os temas das reuniões nas USFs incluíssem não só aspectos relativos ao processo de trabalho - como o trabalho interdisciplinar e o relacionamento interpessoal entre os profissionais - mas também os princípios doutrinários e organizativos do SUS, as ferramentas de trabalho na ESF, alguns aspectos epidemiológicos, além de capacitação técnica sobre temas concernentes ao cotidiano das unidades.

\section{Educação permanente como dispositivo de mudança do processo de trabalho em Teresópolis}

No conjunto das características da ESF, o trabalho em equipe é destacado como um dos pressupostos mais importantes para a reorganização do processo de trabalho e como possibilidade de uma abordagem mais integral e resolutiva (Brasil, 1997, 2001). A integralidade do cuidado depende da integração do sistema, e esta só é possível quando se estabelecem relações de parcerias e de corresponsabilidades entre as equipes de saúde, relações que resultam, principalmente, do envolvimento dos trabalhadores de saúde e da compreensão de que nenhum profissional ou equipe domina sozinho todos 
os conhecimentos necessários para a produção de cuidado integral (Dias, Bertolini e Pimenta, 2011).

De acordo com Ceccim e Ferla,

Uma instituição se faz de pessoas, pessoas se fazem em coletivos e ambos fazem a instituição. Todos e cada um dos profissionais de saúde trabalhando no SUS, na atenção e na gestão do sistema, têm ideias, conceitos e concepções acerca da saúde e da sua produção; do sistema de saúde, de sua operação e do papel que cada profissional e cada unidade deve cumprir na organização das práticas de saúde. É a partir dessas concepções que cada profissional se integra às equipes ou agrupamentos de profissionais em cada ponto do sistema. É a partir dessas concepções, mediadas pela organização dos serviços e do sistema, que cada profissional opera (Ceccim e Ferla, 2008, p. 164).

Assim, para produzir mudanças de práticas de gestão e de atenção, é fundamental dialogar com as práticas e concepções vigentes, problematizá-las não em abstrato, mas no concreto do trabalho de cada equipe, e construir novos pactos de convivência e práticas que aproximem o SUS da atenção integral à saúde (Ceccim e Ferla, 2008).

Todas as entrevistadas apontaram como mudança no processo de trabalho, ocasionada pela prática da EPS nas USFs de Teresópolis, a melhora nas relações interpessoais nas equipes.

Outra modificação importante foi o retorno da prática das reuniões de equipe para a organização do trabalho, para o compartilhamento de informações técnicas e direcionamentos da SMS e para o planejamento e a avaliação regular das atividades das equipes - buscando adequar o serviço prestado pela USF à realidade das comunidades.

Silva e Trad (2005) perceberam que muitas vezes a reunião semanal de uma equipe da ESF se restringe à socialização do que foi programado individualmente pelos profissionais de nível superior, sendo que os demais membros, especialmente os agentes comunitários de saúde (ACSs), não se sentem à vontade para opinar. No entanto, conforme os relatos nas entrevistas e nas atas das reuniões das facilitadoras, as reuniões nas USFs contaram com a participação ativa dos ACSs, com consequente valorização do seu trabalho, relação de pertencimento às equipes e melhora de sua integração com os alunos da Unifeso.

Houve ainda melhora na relação dos alunos com as equipes das USFs como um todo. Não só porque as reuniões de EPS se constituíram em um espaço que possibilitou o rearranjo de algumas atividades do cotidiano das unidades, dando oportunidade para esses alunos, mas também porque possibilitou a verbalização das queixas das equipes e dos alunos, tornando viável a negociação de espaços. 
Em algumas unidades, houve a formação de 'grupos de educação em saúde' - tais como grupos de hipertensos e diabéticos, grupos de gestantes, grupos de adolescentes -, também considerada pelas facilitadoras uma modificação no processo de trabalho, uma vez que introduziu uma nova atividade no cotidiano das equipes e permitiu o protagonismo dos profissionais envolvidos.

Em conformidade com a política do ministério, de promover transformações das práticas técnicas e sociais nas reuniões de EPS, as facilitadoras ensinaram as equipes a trabalharem com os dados de saúde, como o Sistema de Informação da Atenção Básica (SIAB), o Sistema de Acompanhamento do Programa de Humanização no Pré-Natal e Nascimento (Sisprenatal) e o Sistema de Cadastramento e Acompanhamento de Hipertensos e Diabéticos (Hiperdia), subsidiando a discussão da importância do papel de cada profissional no atendimento aos pacientes e instrumentalizando a busca ativa dos casos nas comunidades.

\section{Educação permanente como prática avaliativa amistosa à integralidade no cotidiano das USFs de Teresópolis}

Historicamente, predominam no Brasil práticas avaliativas pouco sistematizadas e articuladas, instituídas verticalmente. Os seus resultados são muitas vezes utilizados em processos decisórios de alocação de recursos ou implementação de programas, sem levar em consideração o cotidiano das equipes de saúde responsáveis pela execução das ações (Pinheiro e Silva Junior, 2008; Tanaka, 2006). Por conseguinte, as equipes de saúde locais têm dificuldades de se apropriar dos processos avaliativos, em razão do desconhecimento de suas abordagens e da sobrecarga de tarefas exigidas pelos próprios programas, principalmente as de coleta de dados.

Nesta pesquisa, percebeu-se que as entrevistadas não estavam familiarizadas com os mecanismos de avaliação que não fossem os formais, estabelecidos pelo MS, pela Secretaria Estadual de Saúde ou pela SMS, nem com a ideia de que a avaliação pudesse ser uma prática do cotidiano dos serviços, realizada pelos próprios profissionais de saúde (não mais implicados apenas na coleta dos dados), visando modificar suas atividades por meio da análise e da reflexão sobre o seu próprio processo de trabalho.

Entretanto, conforme analisaremos a seguir, quando questionadas sobre as modificações no processo de trabalho das equipes desencadeadas pelas reuniões com as facilitadoras, as entrevistadas pontuaram aspectos que levaram à conclusão de que a prática da EPS no município de Teresópolis cumpriu os atributos necessários para ser considerada uma prática avaliativa amistosa à integralidade. 
Ao congregarem a participação dos trabalhadores e dos usuários, além dos alunos de graduação, as reuniões das facilitadoras nas USFs - e destas com a coordenação, na presença de representantes da gestão da SMS de Teresópolis e de coordenadores das graduações da Unifeso - podem ser consideradas como tendo promovido interações democráticas entre os atores em suas práticas cotidianas - bem como inserido os usuários na adequação entre a demanda e a oferta da produção do cuidado nas unidades.

A criação de grupos de educação em saúde e a capacitação para lidar com os dados de saúde coletados em suas próprias unidades - subsidiando a discussão da importância do papel de cada profissional no atendimento aos pacientes e instrumentalizando a busca ativa dos casos e as visitas domiciliares nas comunidades - são exemplos da garantia da inclusão dos usuários na definição de suas necessidades e na tomada de decisão sobre a oferta de alternativas de cuidado.

$\mathrm{O}$ retorno à realização das reuniões de equipe semanais, com inclusão e valorização dos ACSs, gerando melhora nas relações interpessoais entre os profissionais e levando ao compartilhamento de informações técnicas e direcionamentos da SMS, ao planejamento e à avaliação regular das atividades das equipes, também pode ser considerada uma mudança gerada pela EPS no processo de trabalho nas unidades, a qual reflete uma interação democrática entre os atores e tem clara potência formativa.

Essa potência formativa da prática de EPS em Teresópolis pode ser igualmente representada pela participação dos alunos de graduação nas reuniões com as facilitadoras, capacitando as equipes e os futuros profissionais de saúde a produzirem conhecimentos advindos da realidade de cada unidade e aplicáveis especificamente a ela - além de elaborarem respostas qualificadas às necessidades e o modo mais adequado de provê-las. Assim, ao retomar as dimensões que permitem reconhecer as práticas avaliativas amistosas à integralidade, acreditamos que a prática da EPS no município de Teresópolis possui os atributos habilitadores tanto da dimensão da gestão e organização dos serviços quanto da dimensão das práticas dos trabalhadores e da dimensão do direito à saúde.

\section{Conclusões e recomendações}

Como exposto na introdução deste artigo, a persistência das práticas hegemônicas e fragmentadas, ao mesmo tempo que representa um desafio, reforça a necessidade da implementação da política de EPS. As práticas tradicionais não dão conta de responder aos desafios atuais, uma vez que há pouca abertura para o diálogo, para a escuta, para a integralidade e para a construção coletiva (Mendonça e Nunes, 2011). 
Não obstante seja necessário e indispensável que as várias instâncias articulem caminhos para a formação de novos profissionais de saúde para o SUS a fim de mudar a realidade atual dos serviços, é fundamental que se possibilite o desenvolvimento/atualização do pessoal que já está no SUS. Batista e Gonçalves (2011) chamam a atenção para a necessidade de que sejam legitimadas propostas direcionadas a um desempenho profissional qualificado e em quantidade suficiente em todos os pontos do país.

Em Teresópolis, embora o caráter formativo da avaliação nas práticas de EPS não tenha ficado evidente para as facilitadoras e, consequentemente, para os trabalhadores das USFs e os usuários, provocou uma aprendizagem a partir da problematização do cotidiano das equipes de saúde da família, não só para os trabalhadores como também para os alunos da graduação inseridos nas unidades.

Acreditamos que, ao integrar os estudantes e os coordenadores da graduação da Unifeso, os gestores da SMS de Teresópolis, os profissionais das equipes da ESF e os usuários das USFs, as reuniões das facilitadoras - tanto aquelas nas unidades quanto as com a coordenação - promoveram mudanças no processo de trabalho das equipes, viabilizaram uma formação crítica e reflexiva dos atuais e futuros profissionais de saúde, fortaleceram a participação social e aproximaram a SMS das questões locais de saúde, mesmo que de forma incipiente.

Quanto aos aspectos de integralidade observados na prática da EPS em Teresópolis, destacamos o estímulo ao diálogo entre gestão, trabalhadores e usuários nas reuniões com a coordenadora e nas USFs; a avaliação do processo de trabalho das equipes da ESF, voltada para a adequação do cuidado à saúde, levando em consideração a realidade local; e a participação dos usuários nas reuniões com as facilitadoras, garantindo sua inclusão na definição de suas necessidades e na tomada de decisão sobre a oferta do cuidado.

Apesar de não ser possível generalizar as conclusões de um estudo de caso, esta pesquisa permitiu olhar os reflexos da PNEPS na ESF de Teresópolis, principalmente no tocante ao seu potencial como prática avaliativa amistosa à integralidade. Nesse sentido, cabe ressaltar a existência de um contexto político particular do município estudado, representado pela parceria de longa data entre a Unifeso e a SMS.

Espera-se que a disseminação deste estudo possa subsidiar outros municípios na implantação da EPS, explorando sua capacidade avaliativa, de modo a contribuir para a consolidação do SUS. Todavia, é fundamental que os PEPS estejam em pleno funcionamento e que fiquem claras as regras para a construção, o financiamento e a execução dos projetos de interesse locorregional relativos à PNEPS.

Por fim, recomenda-se pensar a EPS além da atenção primária, perpassando todos os níveis de atenção em saúde - em direção não só a um 
serviço, mas a um sistema de saúde humanizado, integral, capaz de autoavaliação e autogestão.

\section{Colaboradores}

O primeiro autor realizou pesquisa de campo e participou de todas as etapas de produção do artigo. A segunda autora participou na condição de orientadora. O terceiro autor colaborou como co-orientador. Os três autores tiveram participação na elaboração do artigo.

Resumen El objetivo de este artículo es discutir el papel de la educación permanente en salud como práctica evaluadora amigable a la integralidad en el quehacer cotidiano de los servicios de salud, además de su influencia en el cambio del proceso de trabajo de los equipos de salud de la familia, tomando como ejemplo la experiencia del municipio de Teresópolis, en Río de Janeiro, Brasil, en 2007 y 2008. Se realizó una investigación cualitativa de enfoque descriptivo. La base teórica se compone de revisión bibliográfica, análisis de documentos oficiales, actas de las reuniones de los facilitadores con la coordinadora del grupo y actas de la Comisión Intergestores Bipartita. El campo de observación consistió en entrevista semiestructurada con el grupo de facilitadores. Para el estudio de las entrevistas y actas, se optó por el análisis de contenido y se efectuó el cotejo de las fuentes como prueba eficiente de validación. Se observó que los profesionales tienen además dificultades para reconocer como herramientas de evaluación instrumentos diferentes de los oficiales. No obstante, en Teresópolis, la práctica de educación permanente fue capaz de fomentar cambios en el proceso de trabajo, permitir la formación crítica y reflexiva de los actuales y futuros profesionales de la salud, fortalecer la participación social y aproximar la gestión de las cuestiones locales de salud, comprobando que puede ser considerada una práctica evaluadora amigable a la integralidad.

Palabras clave proceso de trabajo; educación permanente en salud; práctica evaluadora amigable a la integralidad. 


\section{Notas}

1 Universidade do Estado do Rio de Janeiro, Departamento de Medicina Integral Familiar e Comunitária, Rio de Janeiro, Brasil.

<tsyamamoto@gmail.com>

Correspondência: Departamento de Medicina Integral, Familiar e Comunitária, Boulevard 28 de Setembro, 77, $2^{\circ}$ andar, CEP 20551-900, Prédio dos Ambulatórios, Vila Isabel, Rio de Janeiro, RJ, Brasil.

2 Universidade Federal Fluminense, Instituto de Saúde Coletiva, Programa de Pós-Graduação em Saúde Coletiva, Departamento de Planejamento em Saúde, Niterói, Rio de Janeiro, Brasil.

$<$ monicatcmachado@gmail.com>

3 Universidade Federal Fluminense, Instituto de Saúde Coletiva, Programa de Pós-Graduação em Saúde Coletiva, Departamento de Planejamento em Saúde, Niterói, Rio de Janeiro, Brasil.

<agsilvaj@gmail.com>

4 Este artigo foi extraído da dissertação de mestrado intitulada Política Nacional de Educação Permanente no SUS: estudo da experiência do Município de Teresópolis/RJ sob a perspectiva dos facilitadores de educação permanente, de Thaís Sayuri Yamamoto, apresentada em 27 de junho de 2011 no Programa de Pós-Graduação em Saúde Coletiva, da Universidade Federal Fluminense.

\section{Referências}

ALVES, Josenira C. R. Politica Nacional de Educação Permanente no SUS: estudo da implementação sob a perspectiva dos gestores da Secretaria Municipal de Saúde de Teresópolis em 2006. 184 fls. Tese (Mestrado em Saúde Coletiva) - Instituto de Medicina Social, Universidade do Estado do Rio de Janeiro, Rio de Janeiro, 2007.

AYRES, José R. C. M. Norma e formação: horizontes filosóficos para as práticas de avaliação no contexto da promoção da saúde. Ciência \& Saúde Coletiva, Rio de Janeiro, v. 9, n. 3, p. 583-592, 2004.

BARDIN, Laurence. Análise de conteúdo. Tradução de Luís Antero Reto e Augusto Pinheiro. Lisboa: Edições 70, 2010, 281 p.
BATISTA, Karina B. C.; GONÇALVES, Otília S. J. Formação dos profissionais de saúde para o SUS: significado e cuidado. Saúde e Sociedade, São Paulo, v. 20, n. 4, p. 884-899, 2011.

BRASIL. Ministério da Saúde. Saúde da Família: uma estratégia para a reorientação do modelo assistencial. Brasília, DF: Coordenação de Saúde da Comunidade, Secretaria de Assistência à Saúde, Ministério da Saúde, 1997.

BRASIL. Ministério da Saúde. Departamento de Atenção Básica. Guia prático do Programa de Saúde da Família. Brasília, DF: Ministério da Saúde, 2001. Disponível em: $<$ http://189.28.128.100/dab/docs/publicacoes/ geral/guia_pratico_saude_familia_psfl.pdf > Acesso em: 21 abr. 2010. 
BRASIL. Politica de Educação e Desenvolvimento para o SUS: caminhos para a educação permanente em saúde. Brasília, DF: Ministério da Saúde, 2004a. Disponível em: $<$ http://bvsms.saude.gov.br/bvs/publicacoes/ politica2_vpdf.pdf>. Acesso em 21 abr. 2010.

BRASIL. Portaria n. 198/GM, de 13 de fevereiro de 2004. Institui a Política Nacional de Educação Permanente em Saúde como estratégia do Sistema Único de Saúde para a formação e o desenvolvimento de trabalhadores para o setor e dá outras providências. Brasília, DF: Ministério da Saúde, 2004b. Disponível em: <www.nescon.medicina.ufmg. br/biblioteca/imagem/1832.pdf > . Acesso em: 21 abr. 2010.

BRASIL. Ministério da Saúde. Portaria n. 1.996, de 20 de agosto de 2007. Dispõe sobre as diretrizes para a implementação da política nacional de educação permanente em saúde. Diário Oficial da União, Poder Executivo, Brasília, DF, 22 ago. 2007. Disponível em: $<$ http://bvsms.saude.gov.br/bvs/saudelegis/ gm/2007/prt1996_20_08_2007.html>. Acesso em: 21 jun. 2010.

BRASIL. Ministério da Saúde. Portaria n. 2.200, de 14 de setembro de 2011. Define recursos financeiros do Ministério da Saúde para a Política Nacional de Educação Permanente em Saúde. Diário Oficial da União, Poder Executivo, Brasília, DF, 15 set. 2011. Disponível em: <http://bvsms.saude.gov.br/bvs/saudelegis/ gm/2011/prt2200_14_09_2011.html > . Acesso em: 21 jun. 2010.

CECCIM, Ricardo B. Educação permanente em saúde: desafio ambicioso e necessário. Interface: Comunicação, Saúde, Educação, Botucatu, v. 9, n. 16, p. 61-177, 2005a.

CECCIM, Ricardo B. Educação permanente em saúde: descentralização e disseminação de capacidade pedagógica na saúde. Ciência \& Saúde Coletiva, Rio de Janeiro, v. 10, n. 4, p. 975-986, 2005b.

CECCIM, Ricardo B.; FERLA, Alcindo A. Educação permanente em saúde. In: PEREIRA, Isabel B. Dicionário da educação profissional em saúde. 2. ed. rev. e ampl. Rio de Janeiro: EPSJV, 2008. p. 162-168.

CECCIM, Ricardo B.; FEUERWERKER, Laura C. M. O quadrilátero da formação para a área da saúde: ensino, gestão, atenção e controle social. Physis, Rio de Janeiro, v. 14, n. 1, p. 41-65, 2004.

COELHO, Juliana S. Construindo a participação social no SUS: um constante repensar em busca de equidade e transformação. Saúde e Sociedade, São Paulo, v. 21, supl. 1, p. 138$151,2012$.

DATASUS. Departamento de Informática do Sistema Único de Saúde [internet]. Cadernos de Informação de Saúde, Teresópolis. 2009. Disponível em: <http://tabnet.datasus.gov.br/ tabdata/cadernos/rj.htm>. Acesso em: 7 jun. 2009.

DIAS, Maria D. do A.; BERTOLINI, Grazielle C. S.; PIMENTA, Aparecida L. Saúde do trabalhador na atenção básica: análise a partir de uma experiência municipal. Trabalho, Educação e Saúde, Rio de Janeiro, v. 9, n. 1, p. 137-148, mar./jun. 2011.

ELLERY, Ana E. L.; BOSI, Maria L. M.; LOIOLA, Francisco A. Integração ensino, pesquisa e serviços em saúde: antecedentes, estratégias e iniciativas. Saúde e Sociedade, São Paulo, v. 22, n. 1, p. 187-198, 2013.

FARIA, Horácio P. et al. Processo de trabalho em saúde. 2. ed. Belo Horizonte: Nescon/UFMG, Coopmed, 2009.

FELISBERTO, Eronildo. Monitoramento e avaliação na atenção básica: novos horizontes. Revista Brasileira de Saúde Materno Infantil, Recife, v. 4, n. 3, p. 317-321, 2004.

FELISBERTO, Eronildo. Da teoria à formulação de uma Política Nacional de Avaliação em Saúde: reabrindo o debate. Ciência \& Saúde Coletiva, Rio de Janeiro, v. 11, n. 3, p. 553-563, 2006.

FREIRE, Paulo. Educação e mudança. 30. ed. Tradução de Moacir Gadotti e Lillian Lopes Martins. Rio de Janeiro: Paz \& Terra, 2007. 
L'ABBATE, Solange. Comunicação e educação: uma prática de saúde. In: VER-SUS Brasil: cadernos de textos. Ministério da Saúde, Secretaria de Gestão do Trabalho e da Educação na Saúde, Departamento de Gestão da Educação na Saúde. Brasília, DF: Ministério da Saúde, 2004. Disponível em: $<$ http://bvsms.saude.gov.br/bvs/publicacoes/ versus_brasil_vivencias_estagios.pdf $>$. Acesso em: 12 jun. 2010.

LINO, Mônica M. et al. Educação permanente dos serviços públicos de saúde de Florianópolis. Trabalho, Educação e Saúde, Rio de Janeiro, v. 7, n. 1, p. 115-136, mar./ jun. 2009.

MASCARENHAS, Mônica T. M. Implementação da atenção básica em saúde no município de Niterói, $R J$ : estudo de caso em unidade básica de saúde tradicional e módulo do Programa Médico de Família. 220 fls. Tese (Doutorado em Saúde Pública) - Escola Nacional de Saúde Pública Sergio Arouca, Fundação Oswaldo Cruz, Rio de Janeiro, 2003.

MENDONÇA, Fernanda F.; NUNES, Elisabete F. P. A. Necessidades e dificuldades de tutores e facilitadores para implementar a política de educação permanente em saúde em um município de grande porte no estado do Paraná, Brasil. Interface: Comunicação, Saúde, Educação, Botucatu, v. 15, n. 38, p. 871-882, jul./set. 2011 .

MINAYO, Maria Cecília S. O desafio do conhecimento: pesquisa qualitativa em saúde. 11. ed. São Paulo: Hucitec, 2008.

PEDUZZI, Marina. Trabalho em equipe. In: ESCOLA POLITÉCNICA DE SAÚDE SÃO JOAQUIM VENÂNCIO E OBSERVATÓRIO DOS TÉCNICOS EM SAÚDE (org.). Dicionário da Educação Profissional em Saúde. Rio de Janeiro: EPSJV, 2006. p. 271-278. Disponível em: <www.epsjv.fiocruz.br/upload/ d/Trabalho_em_Equipe_ts.pdf $>$. Acesso em 21 abr. 2010

PEDUZZI, Marina et al. Atividades educativas de trabalhadores na atenção primária: concepções de educação permanente e de educação continuada em saúde presentes no cotidiano de unidades básicas de saúde em São Paulo. Interface: Comunicação, Saúde, Educação, Botucatu, v. 13, n. 30, p. 121 134,2009

PEDUZZI, Marina; SCHRAIBER, Lilia B. Processo de trabalho em saúde. In: PEREIRA, Isabel B; LIMA, Julio C. F. (org.). Dicionário da educação profissional em saúde. 2. ed. rev. ampl. Rio de Janeiro: EPSJV, 2008. p. 320-328.

PINHEIRO, Roseni. As práticas do cotidiano na relação oferta e demanda dos serviços de saúde: um campo de estudo e construção da integralidade. In: PINHEIRO, Roseni; MATTOS, Ruben A. (Orgs.). Os sentidos da integralidade na atenção e no cuidado à saúde. Rio de Janeiro: IMS-Uerj, 2001. p. 67-113.

PINHEIRO, Roseni; SILVA JUNIOR, Aluísio G. Práticas avaliativas e as mediações com a integralidade na saúde: uma proposta para estudos de processos avaliativos na atenção básica. In: PINHEIRO, Roseni; SILVA JÚNIOR, Aluísio G.; MATTOS, Ruben A. (org.). Atenção básica e integralidade: contribuições para estudos de práticas avaliativas em saúde. Rio de Janeiro: Cepesc, IMS/Uerj, Abrasco, 2008. p. 17-41.

PINHEIRO, Roseni; SILVA JUNIOR, Aluísio G. Práticas avaliativas na atenção básica na saúde no estado do Rio de Janeiro: um estudo sobre sistema de controle e monitoramento de ações de saúde. Relatório de pesquisa. Rio de Janeiro, 2009.

PINTO, Vicente P. T. et al. Análise do processo de educação permanente para profissionais do SUS: a experiência de Sobral-CE. Revista de Politicas Públicas (Sanare), Sobral, v. 7, n. 2, p. 62-70, 2008.

SILVA, Jaqueline A. M.; PEDUZZI, Marina. Educação no trabalho na atenção primária à saúde: interfaces entre a educação permanente em saúde e o agir comunicativo. Saúde e Sociedade, São Paulo, v. 20, n. 4, p. 1.018-1.032, 2011.

SILVA, Iêda Z. Q. J.; TRAD, Leny A. B. O trabalho em equipe no PSF: investigando a 
articulação técnica e a interação entre os profissionais, Interface: Comunicação, Saúde, Educação, Botucatu, v. 9, n. 16, p. 25-38, set. 2004/fev. 2005. Disponível em: <www. scielo.br/pdf/icse/v9n16/v9n16a03.pdf $>$. Acesso em: 22 jun. 2010.

SILVA JUNIOR, Aluísio G.; MASCARENHAS, Mônica T. M. Avaliação da atenção básica em saúde sob a ótica da integralidade: aspectos conceituais e metodológicos. In: PINHEIRO, Roseni; MATTOS, Ruben A. (org.). Cuidado: as fronteiras da integralidade. Rio de Janeiro: IMS/Uerj, 2004. p. 241-257.

SILVA, Valéria M. N. O caráter formativo da avaliação nas práticas avaliativas da gestão da atenção básica de Piraí/RJ: o cotidiano dos atores na perspectiva da integralidade. 136 fls. Dissertação (Mestrado em Saúde Coletiva) - Universidade Federal Fluminense, Niterói, 2010.

TANAKA, Oswaldo Y. Caminhos alternativos para a institucionalização da avaliação em saúde. Ciência \& Saúde Coletiva, Rio de Janeiro, v. 11, n. 3, p. 571-572, 2006.
VIANNA, Maria L. T. W.; CAVALCANTI, Maria L.; PINA CABRAL, Marta. Participação em saúde: do que estamos falando? Sociologias, Porto Alegre, n. 21, p. 218-251, 2009.

YAMAMOTO, Thaís S. Politica Nacional de Educação Permanente no SUS: estudo da experiência do município de Teresópolis/RJ sob a perspectiva dos facilitadores de educação permanente. 68 fls. Dissertação (Mestrado em Saúde Coletiva) - Universidade Federal Fluminense, Niterói, 2011. Disponível em: <www.uff.br/saudecoletiva/images/ Documentos/dissertacoes/disserta $\%$ C $3 \%$ A7\%C3\%B5es \% 202011/Disserta \%C3\% A $7 \%$ C $3 \%$ A 30 \% 20Tha $\%$ C $3 \%$ Ads $\% 20$ Sayuri\%20Yamamoto.pdf $>$. Acesso em: 3 fev. 2013.

YIN, Robert K. Estudo de caso: planejamento e métodos. 3. ed. Tradução de Daniel Grassi. Porto Alegre: Bookman, 2005.

Recebido em 10/07/2013

Aprovado em 11/02/2014 